\title{
An evaluation of support assets in addiction recovery settings in Kenya
}

\author{
Elias Kinoti Kithuri ${ }^{1 *}$, Ronald Harvey ${ }^{2 *}$ and Leonard A. Jason ${ }^{3}$ \\ ${ }^{1}$ Meru University of Science and Technology, Meru, Kenya \\ ${ }^{2}$ American University in Bulgaria, Blagoevgrad, Bulgaria \\ ${ }^{3}$ DePaul University, Chicago, IL, USA
}

\begin{abstract}
The purpose of this study was to identify and contrast the social support assets (family, neighborhood, school, community, general and abstinence-specific social support, social networks, and religious congregations) used by individuals in three types of substance abuse treatment settings in Kenya. The three types of settings were treatment centers using professional therapeutic staff, peer-led residential recovery settings, and home-based care with families. Specifically, we compared addiction severity and other problems using the Addiction Severity Index-Lite, the use of social networks using the Important People Inventory, the individual and community assets using the Asset Based Community Development scale, and perceived control using the Alcoholics Anonymous Intention Measure. Results indicated that there were few differences between settings in terms of treatment needs as measured by addiction severity or intentions to seek help. Participants in all three settings relied primarily on family and friends in their social networks reflecting the collectivistic Kenyan culture. Participants' behavioral control was related positivity to friends and family support for recovery. The implications of these findings are discussed.
\end{abstract}

\section{An evaluation of support assets in addiction recovery settings in Kenya}

In Kenya, substance use disorders (SUDs) receive fewer resources in national health care planning and funding because physical illnesses such as HIV/AIDS, malaria, and malnutrition take funding priority [1]. The lack of funding and treatment for SUDs represent an urgent developmental and public health concern in Kenya because SUDs primarily affect younger men, a demographic category that is critical for economic development $[2,3]$.

Substance abuse treatment settings do exist in Kenya, but a number of barriers exist for Kenyans seeking treatment. First, most professionally-run substance abuse treatment centers are located in urban areas; however, people in Kenya live in rural areas, where distance and cost of treatment in urban areas are prohibitive. Secondly, substance abuse and mental health awareness, literacy, and treatment are not mainstream, and therefore, are poorly integrated into the health care system, if at all [4]. Thirdly, SUD treatments are relatively new and unknown compared to the Western world. Kenya's oldest substance abuse rehabilitation center, Asumbi Treatment Centre, started in 1978 . Because SUDs and treatment are unfamiliar, Kenyans are often stigmatized; local descriptions of mental health and substance abuse disorders still include such derogatory language as "mad" and "crazy" [5]. Kenya's only national mental health hospital reflects this thinking. It is called Mathari Hospital - "Mathari" meaning a place for those with physical, social, psychological, or moral abnormalities. In Kenyan collectivistic culture, individuals are seen as having broken taboos and social norms and are seen as morally problematic and dangerous, and these shared perceptions may lead to social exclusion [6].

Given these barriers, many Kenyans avoid professional treatment altogether and choose to seek help in the privacy of family or the larger community. This approach is in line with Kretzmann and McKnight's (1993) proposed an alternative to the professional, needs-based service delivery model, called the Asset Based Community Development (ABCD) model. The ABCD approach suggest that community programs are best built on local capacities, skills, and assets rather than relying on external resources that might be unstable. Local assets can support community health and well-being and include resources and interactions with family, neighborhood, school, group organizations, and religious congregations. For instance, Kretzman and McKnight (1993) reveal that the ABCD approach identifies a set of environmental and intrapersonal strengths known to enhance educational and health outcomes. In a number of studies, Benson (1997, 2002, and 2003) demonstrated that communities have multiple pathways that enhance asset-building capacities at the individual, family, and community levels. These assets provide environmental and psychological strengths that enhance the health of a people in need within a community.

There are three core characteristics integral to the ABCD approach by which local communities create successful healthcare systems [7]. First, there are collective community assets which include human capital, natural resources, financial and physical assets, social structures, and cultural assets. Second, ABCD assets are a viable and sustainable instrument of development because they are treated as cultural artifacts passed on to future generations which tend to gain strength within the

Correspondence to: Ronald Harvey, American University in Bulgaria, Blagoevgrad, Bulgaria, Email: rharvey@aubg.edu

Kinoti E. Kithuri, Meru University of Science and Technology, Meru, Kenya, Email: keynotes1972@yahoo.com.

Received: June 12, 2015; Accepted: September 23, 2016; Published: September 28,2016 
community over time. The policies and programs that the community develops builds the long-term health and well-being of the community. Third, the ABCD approach is collaborative; community members are the principal agents of development, so that the people work with government, private, and not-for-profit sectors to fulfill community goals with local resources.

\section{Assets and needs specific to substance abuse}

Substance use disorders tend to progress in severity over time, starting with hazardous and intrusive stages of use, to more severe substance abuse and substance dependence [8]. Individuals, families, and communities play different roles as protective from or enabling SUDs during this progression. Therefore, SUD treatment and recovery health care delivery are at their best when they supply assets that address the developmental stages of SUDs. Zastrow and Kirst-Ashman [9] found that treatment practices have better outcomes when they are applied in a lifespan context. Treatment applications within the context of family, organizational, and community systems have greater impact at the different lifespan stages with an emphasis on strengths and assets.

Four kinds of settings for substance abuse treatments are: (1) professionally-staffed inpatient and/or outpatient medical/chemical dependency treatment programs that use of methadone maintenance for opiates, (2) professional and peer-led residential therapeutic communities that included behavioral changes as well as treatment services, (3) peer-based recovery programs that work are based on peer groups or self-help groups such as Alcoholics Anonymous (AA), and (4) forms of peer-run residential settings such as half-way houses [8]. Each of these settings provides treatment assets to address the specific needs of their clients. For example, in the United States, there are a number of non-professional settings designed to address the needs of people recovering from SUDs, such as AA mutual help groups or Smart Recovery [10]. Self-help in general is a process of giving and receiving non-professional, non-clinical assistance to achieve long-term recovery from alcohol and/or other drug related problems. In self-help groups, experiential credentials form the bases for shared recovery support systems. The governance of self-help also varies in span and degree of peer control in which there is some peer-owned, peer-directed, and peer-delivered structures of governance. These groups provide assets to aid the in recovery of SUDs, such as a recovery community, general and abstinence-specific social support networks.

Similarly, formal and informal systems of care for treatment for SUDs do exist in Kenya. However, little is known about these settings, the reasons why Kenyan clients with SUDs choose a setting to receive treatment, the client characteristics in these settings, and what their experiences are in various kinds of treatment settings. The purpose of this study is to evaluate and contrast the individual characteristics and assets available to individuals in three types of substance abuse treatment and recovery settings commonly used in Kenya: pay-forservice treatment centers using professional therapeutic staff, a free, peer-led residential recovery setting, and self-directed home-based family settings. As an exploratory study of recovery assets in Kenya, we had no specific hypotheses. We were interested in determining if there were differences in substance abuse severity, supports and assets by settings, and whether a number of the support variables would be related to perceptions of behavioral control. We also wanted to identify the major assets available in different treatment and recovery settings using the $\mathrm{ABCD}$ indicators.

We tested whether addiction severity influenced where individuals go for treatment, and whether the various kinds of settings affected perceptions of assets available to people in various recovery settings. First, we looked for differences in addiction severity scores for clients in each setting. Second, we looked for differences in participants' individual assets. Individual assets were operationalized as the intention to seek help as measured by the Alcoholics Anonymous Intention Measure (AAIM). We also examined participants' self-control as measured by their ability to overcome obstacles through the perceived control component of the AAIM. Third, we examined social support assets from friends and family according to living arrangements in each of the three treatment settings.

\section{Procedure}

\section{Participants}

The study used targeted convenience sampling. The first author, a native Kenyan, consulted a list or treatment and recovery settings from The National Authority for the Campaign Against Alcohol and Drug Abuse (NACADA))directory. These provided the contact information for the paid-for professional settings, the peer-led settings, and selfhelp groups (such as AA) by region. The first author telephoned the treatment and recovery centers in Kenya listed in NACADA directory, introduced himself and the purpose of his inquiry, and, if agreed by the site, requested a time and date to visit to introduce himself and the study in detail. If agreed to after meeting with the staff, a meeting with residents as a group was arranged. Potential participants usually gathered in a group at a meeting place at the treatment and recovery settings. After an introduction of the study and a question and answer session, informed consent forms were distributed and another question and answer session was give. If participants agreed, they signed the informed consent and, if they had time, were given the study questionnaires, which took 45 minutes to complete. If participants wanted to complete the questionnaires at another time, a followup visit was arranged. For the home-based care group, the author contacted a self-help group center and asked leaders of these centered to distributed information and the author's contact information. In this case, the study's purpose, informed consent, and data was obtained outside the self-help group center or in the participant's home. In all, we collected data from treatment programs or recovery settings in 10 geographical regions in Kenya.

\section{Settings}

There were eight different professional settings. We defined professional settings as those which offered paid services employing a staff trained in SUD treatment and were not peer-run. Each of the eight programs featured 3-month inpatient residential programs with a variety of individual and group therapies offered within a structured environment. Those entering the program with severe addiction problems are first given supervised detoxification treatments. In addition to a variety of group and individual therapies, the professional settings promoted self-help group attendance.

Most of the 26 participants in the home-based care condition had been in SUD treatment programs prior and were currently living with their families or on their own. There were only 3 people in this condition that had not previously been in some kind of treatment program (i.e. these individuals who quit on their own).

Those in the peer-led residential recovery settings came from two settings: the U-Turn for Christ Center, and the Omar Project. Both programs provide clients with services at no cost in exchange for performing work at the facilities. The U-Turn for Christ center is 
a residential, Christian faith-based substance abuse recovery setting that includes a self-help managed program that provides both housing and jobs. This center focuses on the faith relationships and encourages residents to shape their lives around the long-term goal of stabilizing oneself through faith in God and values of Christian living. Their program has two phases. During phase one, residents are provided housing for a minimum of two months in a large community farm and attached dormitory housing. While in phase one, residents have time to attend morning religious devotional activities, complete daily chores, engage in volunteer services and devote time to personal reflection during the evenings. They also go to self-help groups in the morning and evening.

Phase two typically lasts six months during which residents are allowed to work on a variety of tasks on the farm, and become a support group mentors for those in the phase one. Some of the farming activities include planting, caring and harvesting various types of vegetable plants and caring for dairy cows. Many of the residents are engaged in church activities with their sponsor the U-Turn Calvary church, which is located on the same grounds as the farm with the dorm/recovery center. Only men can enroll in these programs, and individuals in both phases of the program live in the same dormitory

Following completion of the program, residents can choose to remain at the center, where they may assume more responsibilities on the farm. The total length of stay in the program is one year after which residents may choose to re-join their families. However, there are some residents who become more involved with the church and stay on to become staff members in the recovery program or with the church. Sister churches and the profits made from the sale of farm vegetables serve as the source of revenue.

The other peer-led residential recovery setting is called the Omar Project, a self-help Islamic faith-based community initiative. The Omar Project has a rented house where only new people come and stay for 3 months and then return to their communities. The Omar Project only houses individuals who are new to recovery and support is provided from others residents who have gone through the program. The major resources of the Omar Project are peer group supports and housing. After about 3 months, most residents go back to their families and become recruiters for the next group of residents in need of recovery. Members of the house attend self-help groups where they have an opportunity to learn life skills, including anger management. The members are all Muslims, and men and women live on separate levels. There is no cost for program participation, as the Omar Project relies on donations from a local mosque. Unlike U-turn for Christ, Omar project does not have a farm as a source of income and food.

\section{Measures}

Addiction Severity Index Lite, 5th Edition (ASI-Lite) was developed by McLellan, Kushner, Metzger, Peters, Smith, Grissom, and Argeriou [11], and it assesses problems in areas commonly affected by substance abuse including: medical status, drug use, alcohol use, illegal activity, family relations, and psychiatric condition. Compared to the full ASI, the ASI-Lite contains 22 fewer questions and does not include some severity ratings or the family history grid. The ASI-Lite records demographic moderator variables (e.g., gender, ethnicity) and status outcome items for employment, criminal involvement, and health status. In each of these areas, participants list the number, extent, and duration of problems or symptoms for lifetime and in the past 30 days.

Alcoholics Anonymous Intention Measure (AAIM) is a 17-item scale, which was developed through utilization of [11] framework of the theory of planned behavior to measure participants' intentions to attend a 12-step group over the next year. The AAIM has a "readiness component" for participation in a 12-step group (e.g. Narcotics Anonymous or Alcoholics Anonymous) [13]. The "attitude component" relates to the expected outcome from 12-step participation, and rates the desirability of each outcome. All the items use a seven-point response Likert scale. The AAIM scale reported good internal consistency $(\alpha=.83)$.

Important People Inventory (IP) was developed by Clifford and Longabaugh [14], and is based on the Important People and Activities Inventory as applied to alcohol and drug recovery. It is intended to measure participants' perceptions of support received from important people in their network: types of support received (general or alcohol/ drug abstinence-specific support), the amount support received from their network, network member's feelings towards participant's recovery, number of contacts with the network, and other people's reactions to the participant not using alcohol or drugs.

One subscale of the IP is general support from people in the network. The participant reported how the IP is generally supportive, sensitive to personal needs, helps think about issues or solve problems, and offers the participant moral support. Positive general social support from friends has been found to be a predictor of lower alcohol use [15-17].

Development Assets Scale is a measure of a community's building blocks, such as family, neighborhood, school, group organizations, and congregations, which are essential for a community's health and wellbeing. $[18,19]$ identified 40 assets and grouped them into categories that influence community members' lives. In this framework, a category of external assets includes support, empowerment, boundaries and expectations, and constructive use of time. The category of internal assets involves relationships and opportunities provided by fellow members of the community, and assets that tap into the competencies and values that are specific to groups. In Oliva, Lopez, and Antolin's [20] validation study, the Development Assets scale showed an overall high internal reliability: $\alpha=.93$, while reliabilities for each of the dimensions were either high or acceptable.

\section{Results}

\section{Demographic variables}

Of the approximately 400 potential participants approached in the three types of settings, a total sample of 222 adults (56\%) agreed to participate. There were participants drawn from professionallymanaged treatment settings $(37.9 \%, \mathrm{n}=83)$, self-run peer managed recovery settings $(50.2 \%, \mathrm{n}=110)$, and from home-based care (living with family) after treatment $(11.9 \%, \mathrm{n}=26)$. The sample consisted of 206 males (92.8\%), and 16 females (7.2\%), age range from 18-68 (M =36.9). We interviewed 90 individuals in the U-Turn for Christ Center and 20 individuals at the Omar Project. The sample demographic characteristics; education, employment status, and marital status can be found in Table 1. Participants reported on average 11.9 years of substance abuse and/or alcoholism, which ranged from 1 year to 40 years of substance abuse.

\section{Main analyses}

We first examined whether there were significant differences in ASI addiction severity between the professional treatment sites, peer managed residential settings, and home-based care. The ASI variables 
Table 1. Demographical Characteristics of the Kenyan sample.

\begin{tabular}{|l|c|c|}
\hline & M & SD \\
\hline Age & 36.9 & 10.50 \\
\hline Length of alcohol-drug abuse (yrs) & & \\
\hline Treatment & 11.88 & 8.95 \\
\hline Self-help & 11.33 & 6.38 \\
\hline Home-based care & 14.60 & 8.03 \\
\hline Length of stay (Months) & & \\
\hline Treatment & 5.61 & 17.98 \\
\hline Self-help & 6.97 & 25.82 \\
\hline Home-based care & 29.33 & 71.99 \\
\hline & $N$ & $\%$ \\
\hline Education (Months) & & \\
\hline High school & 55 & 24.9 \\
\hline College & 137 & 62.1 \\
\hline Vocation school & 4 & 1.8 \\
\hline Form 5-6th & 7 & 3.2 \\
\hline Marital Status & & \\
\hline Married/Partnered & 87 & 39.6 \\
\hline Separated/Divorced/Widowed & 37 & 16.8 \\
\hline Single & 96 & 43.2 \\
\hline Other & 2 & .9 \\
\hline Employment & 145 & 65.3 \\
\hline Full time/Part-time/Self-employed & 49 & 21.7 \\
\hline Unemployed/disability & 3 & 11.2 \\
\hline Student & & 1.4 \\
\hline Retired & & \\
\hline
\end{tabular}

used in this study were: alcohol use $(\mathrm{M}=.36, \mathrm{SD}=.14)$, Family status $(\mathrm{M}=.36, \mathrm{SD}=.12)$, Legal status $(\mathrm{M}=.34, \mathrm{SD}=.14)$, psychiatric severity $(\mathrm{M}=.25, \mathrm{SD}=.16)$, drug use $(\mathrm{M}=.11, \mathrm{SD}=.07)$ and medical status $(\mathrm{M}$ $=.08, \mathrm{SD}=.16$ ). For the ASI alcohol and drugs severity scores (both past 30 days and lifetime), there were no statistically significant differences $\left[F(2,214)=.019, p=.454, \eta^{2}=.007\right]$.

We next examined Alcoholics Anonymous intentions scores as measured on the AAIM. There was again no statistically significant effect of across professional treatment sites, peer managed residential settings, and home-based care $\left[F(2,210)=1.796, \mathrm{p}=.168, \eta^{2}=.017\right]$. Perceived control was used as an outcome variable because of its relevance to taking action to resist substance use. There were again no statistically significant effect of perceived control across the three settings $\left[F(2,210)=1.796, \mathrm{p}=.168, \eta^{2}=.017\right]$.

For the Important People Inventory (IP), there were no significant differences across professional treatment sites, peer managed residential settings, and home-based care for frequency of contact with the network in the last six months $\left[F(2,209)=.055, p=.947, \eta^{2}=.001\right]$ or the type of social support received from their social network $[F(2$, $\left.211)=.378, \mathrm{p}=.685, \eta^{2}=.004\right]$. However, the peer managed residential settings had a marginal statistical difference regarding how participants experienced their network's social support in the form of attitudes about self-help groups $\left[F(1,211)=2.219, \mathrm{p}=.055 \eta^{2}=.027\right]$. The means for the three groups were: professional treatment $(\mathrm{M}=5.5, \mathrm{SD}=.8$ ), peer managed residential settings $(\mathrm{M}=5.6, \mathrm{SD}=.0 .9)$, and home-based care groups $(\mathrm{M}=5.8, \mathrm{SD}=1.0)$. Using the Tukey test, people in homebased care experienced significantly higher IP social support from their network compared to the other two conditions. There were no statistical significant setting differences for asset based community level scores, $F(2,208)=.641, p=.528, \eta^{2}=.006$.
In the current study, people in treatment and recovery settings in Kenya reported high levels of social support $(\mathrm{M}=4.54, \mathrm{SD}=1.17)$, attachment to their neighborhood, $(M=4.29, \mathrm{SD} 1.78)$, feeling secure in their neighborhood $(\mathrm{M}=4.27, \mathrm{SD}=1.37)$, having available activities to take part in $(\mathrm{M}=4.21, \mathrm{SD}=1.84)$, and experiencing empowering support $(\mathrm{M}=3.64, \mathrm{SD}=1.56)$. There was no statistical significant group difference between professional treatment sites, peer managed residential settings, and home-based care for asset-based community level scores $\left(F(2,208)=.641, p=.528, \eta^{2}=.006\right)$. The support and empowerment scale showed reliability of $\alpha=.91$, attachment to the neighborhood, $\alpha=.91$, security, $\alpha=.87$, social control, $\alpha=.85$, and activities $\alpha=.80$.

\section{Secondary analyses: Perceived control}

Multiple regressions were performed to investigate whether IP social support for alcohol-drug abstinence significantly predicted participant's perception of their behavioral control. Behavioral control is the ability for people in recovery to achieve their specific recovery goals in the face of everyday obstacles. A significant model emerged, $F(7,191)=3.78, p<.001$. The model accounted for $8.9 \%$ of the variance (Adjusted $R^{2}=.089$ ) and demonstrated that the degree of social support provided by the network significantly predicted participants perceptions of behavioral control $(\beta=.19, p<.05)$. Hence, increases in the participant's behavioral control correlated with a corresponding increase in degree of network support for alcohol-drug abstinence. In addition, the network support for being in peer-managed settings was statistically significant $(\beta=.22, p<.05)$. That is, increases in the network social support in the form of emotional assets supplied towards participants' being in the peer-managed recovery settings condition were associated with increases in participants' perceptions of behavioral control.

\section{Discussion}

In this study, professional, peer-led, and home-based settings appeared to have similarly high access to social network assets used as a recovery resource. We also found no significant group differences in participants' experiences of community assets, such as neighborhoods, local institutions, and schools and religious settings. Supports received from extended family structures in the community are well-founded in Kenya's cultural context. According to Moos [21], the best circumstance to achieve long-term recovery is to create models that address clients' life contexts. These models may emerge deliberately via program design, or organically out of clients' needs and available assets. The fact that relatively high and positive scores across a range of variables suggests that each of these settings had useful contributions to the care of individuals with substance abuse in Kenya.

The importance of positive social support for recovery was reflected in the positive relationship between alcohol-drug abstinence social support and perceived behavioral control. The more positive social support, the more likely participants believed they were in control of their behaviors. Although the significance was marginal, treatmentseekers in Kenyan collectivistic cultures preferred home-based care among friends and family for support over strangers in professional and peer-led settings. This implies that, regardless of available resources to pay for services, people seeking treatment from SUDs in Kenya rely on their community members to support their recovery.

This study's major limitations are its cross-sectional design and the self-selection bias which likely resulted in measurement insensitivity and ceiling effects across the settings. For example, any positive social 
support might have simply reinforced the notion that participants made the best choice for their situation. In addition, none of the measures used in this study with the exception of the ASI have been used in Kenyan context. Future studies should select instruments that have been thoroughly tested to capture variability in more collectivistic Kenyan populations, and to use a random assignment design. Another limitation of this study is that it lacked a heterogeneous population, as the majority of the participants of this study were males. Future studies should include females to compare women's substance use patterns to males, and if there are gender-specific barriers to recovery. Given that stigmatization is likely higher among Kenyan women than men for SUDs, future research should specifically measure stigmatization to determine how these factors into support assets needed in recovery.

Currently, Kenya's treatment and recovery programs are all modeled on Western programs and are in a formative stage. The entry of treatment and recovery medicine and mental health issues requires new cultural perspectives. Local cultural perspectives on mental health and substance abuse are based on moral assumptions, with ideas such as substance abuse results from an individual's moral problem. People with SUDs will tend to hide their condition from others and are less likely to disclose any information about their problems even to health care institutions and professionals. Therefore, it is vitally important for future researchers further explore the challenges for Kenyans in SUD recovery in a predominantly collectivistic culture. Individualbased processes such as self-concept, self-esteem, self-mastery and decision-making are compounded by community expectations, which take precedence over the individual. Individual choices are constrained by interwoven dependence on the group judgment as opposed to individual competency and decisions. In these circumstances, an individual is constrained to act upon a behavior that is socially acceptable and fits into the community's expectations. The tension is doing what is culturally acceptable can be tapped to create systems that support SUD recovery.

This may explain why most people preferred home-based care. In our study, the majority of social support networks for people in recovery and treatment in Kenya had a structure largely dominated by family members: $25.2 \%$ were family, wife, or children, whereas $30.5 \%$ were parents and siblings. This aligns with participants' reporting that $67.1 \%$ of them were referred to treatment and recovery setting by a family member. This finding indicates the important role of family in a collectivist culture both as a resource for the individual in treatment, but also encouraging recovery to benefit the collective group. Participants' level of stigma was not measured, but some research states that stigma that prompted participants to prefer home-based care because it is less stressful [22]. Still, it is likely that family members could benefit from information and education about SUDs to enhance their understanding and support for relatives and friends with substance abuse problems. Future studies should focus on home- and self-help based interventions and the impact of community education for improving interventions. This could encourage healthcare policy makers in Kenya to create community-run and community-based settings to support homebased care. Such systems are likely cheaper, easier to replicate, and more sustainable than more expensive professional care settings [23].

This study has two unique characteristics. First, most studies in Kenya on substance abuse are of school- and hospital-based systems [24]. This study examined the relatively new phenomenon of peer-run treatment that are less than ten years old, and the older traditional model of home-based care in the context of the Kenyan mental healthsubstance abuse system. Secondly, we used and assets-strengths model as opposed to the deficits-needs model. Although Kenyan treatment for addiction is still in formative stages, we agree with [21]: systems of care need to consider the client's views about self, body, mind, relationships and spirit, in the context of local cultural and environmental factors to achieve lasting changes. Recovery programs in Kenya are most effective when they provide support from peers and allies, helping those in recovery return to an active role in their communities.

\section{References}

1. Walt LC, Kinoti E, Jason LA (2013) Industrialization stresses, alcohol abuse and substance dependence: Differential gender effects in a Kenyan rural farming community. Int J Ment Health Addict 11: 369-380. [Crossref]

2. Whiteford HA, Degenhardt L, Rehm J, Baxter AJ, Ferrari AJ, et al. (2013) Global burden of disease attributable to mental and substance use disorders: findings from the Global Burden of Disease Study 2010. Lancet 382: 1575-1586. [Crossref]

3. Singer M (2008) Drugs and development: the global impact of drug use and trafficking on social and economic development. Int J Drug Policy 19: 467-478. [Crossref]

4. Room R (1998) Mutual help movements for alcohol problems in an international perspective. Addiction Research 6: 131-145.

5. Kinoti KE, Jason LA, Harper GW (2011) Determinants of Alcohol Khat and Bhang Use in Rural Kenya. Afr J Drug Alcohol Stud 10: 107-118. [Crossref]

6. Hofstede G (1980) Culture's consequences: Comparing values behaviors institutions and organizations across nations. Beverly Hills CA: Sage.

7. Cunningham G, Mathie A (2002) Asset-based community development: An overview. Coady International Institute.

8. Borkman JR, Kaskutas LA, Room J, Barrows D (1998) An historical and developmental analysis of social model programs. Journal of Substance Abuse Treatment 15: 17-17. [Crossref]

9. Zastrow C, Kirst-Ashman KK (2007) Understanding human behavior and the social environment (7th edition) Belmont California: Thompson Books.

10. White WL (2004) Addiction recovery mutual aid groups: An enduring internationa phenomenon. Addiction 99: 532-538.

11. McLellan AT, Kushner H, Metzger D, Peters R, Smith I, et al. (1992) The Fifth Edition of the Addiction Severity Index. J Subst Abuse Treat 9: 199-213. [Crossref]

12. Ajzen I (2002) Constructing a TPB questionnaire: Conceptual and methodologica considerations. University of Amherst, Amhest. http://people.umass.edu/aizen/pdf/tpb. measurement.pdf

13. Zemore E, Sahara KA Lee (2010) Devlopement and validation of the alcoholic anonymous imntention measure (AAIM). Drug Alcohol Depend 104: 204-211. [Crossref]

14. Clifford PR, Longabaugh R (1991) Manual for the Administration of the Important People and Activities Instrument. Adapted for use by Project MATCH for NIAAA 5R01AA06698-05 Environmental Treatment of Alcohol Abusers, Richard Longabaugh, Principal Investigator.

15. Groh D. R, Jason LA, Davis MI, Olson BD, Ferrari JR, et al. (2007) Friends, family and alcohol abuse: An examination of general and alcohol-specific social support. The American Journal on Addictions 16: 49-55. [Crossref]

16. Falkin GP, Strauss SM (2003) Social supporters and drug use enablers: a dilemma for women in recovery. Addict Behav 28: 141-155.

17. Longabaugh R, Beattie M, Noel N, Stout R, Malloy P (1993) The effect of social investment on treatment outcome. J Stud Alcohol 54: 465-478. [Crossref]

18. Benson P (1996) Developmental assets among Minneapolis youth: The urgency of promoting healthy community. Minneapolis.

19. Benson PL, Leffert N, Scales PC, Blyth DA (1998) Beyond the "village' rhetoric" Creating healthy communities for children and adolescents. Applied Developmental Science 2: 138-159.

20. Oliva A, Antolin L, Lopez AM (2011) Development and validation of a scale for the measurements of adolescents' development in the neighborhood. Social Indicators Research 106: 563-576.

21. Moos RH (2003) Social contexts: transcending their power and their fragility. American Journal of Community Psychology 31: (1-2) 1-13. [Crossref] 
22. Chun C, Moos RH, Cronkite RC (2006) Culture: A fundamental context for the stress and coping paradigm. (pp 29-53) in Wong PT, LC Wong LC (Eds.) Handbook of multicultural perspectives on stress and coping. Springer Science and Business Media, New York.

23. Lo Sasso AT, Byro E, Jason LA, Ferrari JR and Olson B et al. (2012) Benefits and costs associated with mutual-help community-based recovery homes: The Oxford House model. Eval Program Plann 35: 47-53. [Crossref]

24. Kinoti KE, Jason LA (2012) The status of mental health and substance abuse recovery programs in Kenya's primary health care system. J Psychological Health 1: 25-55.

Copyright: (C2016 Kithuri EK. This is an open-access article distributed under the terms of the Creative Commons Attribution License, which permits unrestricted use, distribution, and reproduction in any medium, provided the original author and source are credited. 